\title{
Antimicrobial Resistance Pattern of Staphylococcus aureus Isolated from Infected Wounds at Sohag University Hospitals.
}

\author{
Alaa El Din Hassan Mohammed, Mona Fattouh Mohamed, Asmaa Mohamed \\ Goda ,Samah Raafat Mohamed.
}

General Surgery Department, Faculty of Medicine, Sohag University, Sohag, Egypt Microbiology Department, Faculty of Medicine, Sohag University, Sohag, Egypt

\section{Abstract}

Background:Staphylococcus aureus is an important human pathogen causing varieties of mild to life threatening community and hospital infections. This study was carried out to determine the antibiotic susceptibility profile of Staphylococcus aureus isolated from infected wounds of patients at Sohag University Hospitals.

patients and methods: 100 clinical isolates of $S$. aureus were obtained from 350 pus samples using standard microbiological techniques. Antimicrobial susceptibility test was performed using the modified Kirby-Bauer disk diffusion method and confirmed by VITEK 2 automated microbiology system. methicillin resistant Staphylococcus aureus (MRSA) detection was also confirmed by detection of mec A gene by PCR.

Results: S.aureus isolates were totally resistant to Penicillin G and highly resistant to Cefoxitin and Oxacillin. They were moderately resistant to Gentamycin, Tetracycline, Erythromycin, Clindamycin, Doxycycline and Chloramphenicol.They showed low resistance to Ciprofloxacin, Levofloxacin Trimethoprim/ Sulfamethoxazole, Moxifloxacin and Azithromycin. All isolates were sensitive to Qunipristin/Dalfopristin, Linezolid, Vancomycin ,Tigecycline, Nitrofurantoin, Rifampicin and Teicoplanin.Among the 100 isolated Staphylococcus isolates, $89(89 \%)$ were identified as MRSA.

Conclusion: The high incidence of S.aureusisolates resistant to the commonly used antibiotics in the hospital calls for urgent need to put in place measures to curtail the spread of $M R S A$ infections in the hospital.

\section{Introduction:}

Staphylococcus aureus are clusterforming, facultative aerobic, Grampositive cocci [1].

It exhibits intrinsic ability to ferment carbohydrates with white to deep yellow pigmentation on solid culture media[2]. Also, this bacterium ferments mannitol which turns Mannitol Salt Agar (MSA) yellow. Another key characteristic of this important human and animal pathogen is the production of deoxyribonuclease (DNase) and catalase enzymes along with production of coagulase protein [1].

S.aureusis an opportunistic pathogen implicated as the most common agent of skin and soft tissue infections. It exists in the nasopharynx, skin, eye, intestine and urogenital tract as normal flora. However, it can breach the skin barriers through the wound or surgical incision and cause infection[3] $S$. aureus is resistant to many antibiotics, starting with penicillin and methicillin, until the most recent, linezolid and daptomycin [4].Resistance mechanisms include enzymatic inactivation of the antibiotic (penicillinase and aminoglycosidemodification enzymes), alteration of the target with decreased affinity for the antibiotic (notable examples being penicillin-binding protein $2 \mathrm{a}$ of methicillin-resistant $S$. aureus and D-

Ala -D-Lac of peptidoglycan precursors of vancomycin-resistant strains), trapping of the antibiotic (for vancomycin and possibly daptomycin) and efflux pumps (fluoroquinolones and tetracycline).Complex genetic arrays (staphylococcal chromosomal cassette mec elements or the vanA operon) have been acquired 
by $S$. aureus through horizontal gene transfer, while resistance to other antibiotics, including some of the most recent ones (e.g., fluoroquinolones, linezolid and daptomycin) have developed through spontaneous mutations and positive selection[5].

Methicillin resistant S. aureus (MRSA) is one of the pathogens posing serious threat to the global health care of patients in both community and hospital settings [6-8]. MRSA causes infections such as skin and wound infections, pneumonia, blood stream infections (BSI), meningitis, toxic shock syndrome (TSS), etc. [6]. The present study therefore aimed at providing baseline information on the occurrence and antibiotic resistance pattern of $S$. aureus isolates from wounds of patients admitted in Sohag University .

\section{Patients and Methods}

Place and time of the study: This study was conducted in Department of Medical Microbiology and Immunology, Sohag Faculty of Medicine, Sohag University Hospitals during the period from April 2016 to April 2018.

Patients: All patients were admitted at Sohag University Hospitals from different surgical departments. All patients had infected wounds caused by Staphylococcus aureus. The overt clinical signs associated with local wound infection are: Discharge of pus, swelling, pain, erythema and local warmth, evidence of surrounding tissue involvement or wound breakdown; wound appears unhealthy or deteriorating, and probing infection to the bone (cellulitis, lymphangitis, osteomyelitis or gangrene) [7].

Ethical consideration: Oral consent were obtained from all cases. Ethical approval was taken from the scientific ethics committee of Sohag Faculty of Medicine, Sohag University.
Isolation and Identification of Bacteria: Pus from infected wounds was collected by sterile disposable cotton swabs. All Staphylococcus aureus isolates were identified by, Gram staining (gram positive cocci in grape like clusters) colony morphology (golden on NA,beta hemolytic on blood agar and caused yellow discoloration on MSA) and conventional biochemical tests (positive catalase and coagulase tests ). Antibiotic sensitivity testingwas done by Disk diffusion method( modified Kirby-Bauer ) and confirmed by Automated Antibiotic sensitivity testing(Vitek 2; bioMerieux, France) system: According to modified KirbyBauer method, Muller Hinton agar was used to test antibiotic disks of $S$. aureus antimicrobial resistance and interpreted as per Clinical and Laboratory Standards Institute guidelines (CLSI 2016). The following antimicrobial agents were tested Penicillin G(10 units), Cefoxitin $(30 \mu \mathrm{g})$, Teicoplanin $\quad(30 \mu \mathrm{g})$ Gentamycin $(10 \mu \mathrm{g})$ Azithromycin $(15 \mu \mathrm{g})$,Erythromycin $(15 \quad \mu \mathrm{g})$ Ciprofloxacin $(5 \mu \mathrm{g}) \quad$ Levofloxacin $(5 \mu \mathrm{g}), \quad$ Doxycycline $\quad(30 \mu \mathrm{g})$, Tetracycline $(15 \mu \mathrm{g})$,Trimethoprimsulfamethoxazole $\quad(1.25 / 23.75 \mu \mathrm{g})$,

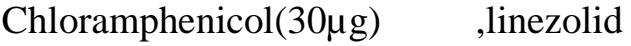
$(30 \mu \mathrm{g})$ and Clindamycin $(2 \mu \mathrm{g})$.

Detection of mec A gene

Simple qualitative polymerase chain reaction for detection of mec $\mathrm{A}$ gene was done as follows:

i. DNA extraction (the boiling method):Few isolated colonies of overnight growth bacteria were suspended thoroughly in distilled water. The suspension was boiled in a water bath, for $10 \mathrm{~min}$. It was centrifuged at $10000 \mathrm{rpm}$ for $5 \mathrm{~min}$, The suspernatant was taken as a template and stored at $-20^{\circ} \mathrm{C}[8]$.

ii. DNA amplification :The amplification reactions were 
prepared in a $25 \mu \mathrm{l}$ volume containing the following; $12.5 \mu \mathrm{l}$ PCR master mix, $7 \mu$ l Sterile Water, $1.25 \mu \mathrm{l}$ forward primer(5'GTG AAG ATA TAC CAA GTG ATT -3'), $1.25 \mu \mathrm{l}$ reverse $\operatorname{primer}\left(5^{\prime}\right.$ ATG CGC TAT AGA TTG AAA GGA $T-3$ ' ) [8] and $3 \mu$ l DNA. The thermal amplification included the following steps: an initial denaturation at $95^{\circ} \mathrm{C}$ for $5 \mathrm{~min} ; 30$ cycles of amplification with $94^{\circ} \mathrm{C}$

\section{Results}

Our study was carried out at Sohag University Hospitals in the period between April 2016 and April 2018. The study included 350 patients with wound infections isolated from patients recruited from different departments. Staphylococcus aureus was isolated in 100 patients .

I. Antibiotic susceptibility profile of the isolated S.aureus strains: S.aureus isolates were totally resistant to Penicillin G and highly resistant to Cefoxitin and Oxacillin. They were moderately resistant to Gentamycin, Tetracycline, Erythromycin, Clindamycin, Doxycycline and Chloramphenicol.They showed low resistance to Ciprofloxacin, Levofloxacin Trimethoprim/ Sulfamethoxazole, Moxifloxacin and Azithromycin. All isolates were sensitive to Qunipristin/Dalfopristin, Linezolid, Vancomycin ,Tigecycline, Nitrofurantoin, Rifampicin and Teicoplanin.Among the 100 isolated Staphylococcus strains, 89(89\%) were identified as MRSA by cefoxitin disc diffusion method (table1)

Table (1):Distribution of the studied patients according to sensitivity pattern to different antibiotics.

\begin{tabular}{|c|c|c|}
\hline Antibiotic & $\begin{array}{l}\text { Sensitive } \\
\text { NO. }(\%)\end{array}$ & $\begin{array}{l}\text { Resistant } \\
\text { NO. }(\%)\end{array}$ \\
\hline Cefoxitin & $11(11.0 \%)$ & $89(89.0 \%)$ \\
\hline PenicillinG & $0(0.0 \%)$ & $100(100 \%)$ \\
\hline Oxacillin & $11(11.0 \%)$ & $89(89.0 \%)$ \\
\hline Gentamycin(CN) & $66(66.0 \%)$ & $34(34.0 \%)$ \\
\hline Ciprofloxacin & $74(74.0 \%)$ & $26(26.0 \%)$ \\
\hline Levofloxacin & $74(74.0 \%)$ & $26(26.0 \%)$ \\
\hline Moxifloxacin & $96(96.0 \%)$ & $4(4.0 \%)$ \\
\hline Erythromycin & $56(56.0 \%)$ & $44(44.0 \%)$ \\
\hline Clindamycin & $62(62.0 \%)$ & 38(38.0\%) \\
\hline Qunipristin/Dalfopristin & $100(100 \%)$ & $\mathbf{0}(\mathbf{0 \%})$ \\
\hline Linezolid & $100(100 \%)$ & $\mathbf{0}(0 \%)$ \\
\hline Vancomycin & $100(100 \%)$ & $\mathbf{0}(0 \%)$ \\
\hline Tetracycline & $42(42.0 \%)$ & $58(58.0 \%)$ \\
\hline Tigecycline & $100(100 \%)$ & $\mathbf{0}(\mathbf{0 \%})$ \\
\hline Nitrofurantoin & $100(100 \%)$ & $\mathbf{0}(\mathbf{0 \%})$ \\
\hline Rifampicin & $100(100 \%)$ & $\mathbf{0}(0 \%)$ \\
\hline Trimethoprim/Sulfamethoxazole & $83(83.0 \%)$ & 17(17.0\%) \\
\hline Teicoplanin & $100(100 \%)$ & $0(0 \%)$ \\
\hline Doxycycline & $42(42.0 \%)$ & $58(58.0 \%)$ \\
\hline Azithromycin & $89(89.0 \%)$ & $11(11.0 \%)$ \\
\hline Chloramphenicol & $56(56.0 \%)$ & $44(4.0 \%)$ \\
\hline
\end{tabular}

II. Distribution of patients according to MRSA and MSSA groups :Among the 100 isolated S.aureus ;89(89\%) were identified as MRSA by cefoxitin disc diffusion 
method confirmed by vitek 2 automated system and by detection of mec A gene by PCR. Mec A gene was present in $89(89 \%)$ of isolates.So,89\% of the S.aureus isolates were $M R S A$ and $11 \%$ were $M S S A$.(figure 1)

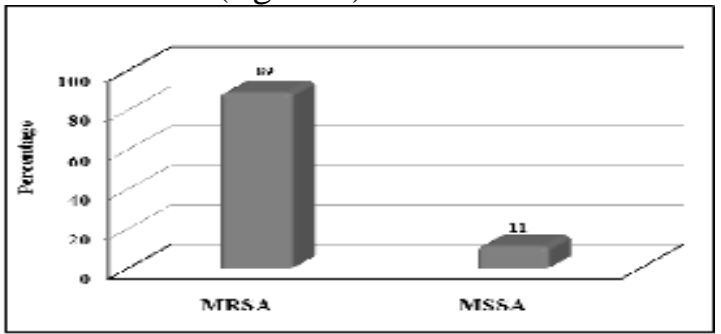

Figure (1): Prevalence of MRSA and MSSA in studied population.

III. Comparison between MRSA and MSSA regarding antibiotic susceptibility profile: All $M R S A$ and $\underline{M S S A}$ isolates were sensitive to Vancomycin, Linezolid, Nitrofurantoin, Rifampicin, Tigecycline Qunipristin/Dalfopristin and Teicoplanin .Also, all MRSA and MSSA isolates were resistant to Penicillin. MRSA showed significantly higher resistance to Gentamycin, Erythromycin and Clindamycin than $\operatorname{MSSA}(\boldsymbol{P}$-value is >0.05). There was no significant difference in susceptibility to Ciprofloxacin ,Levofloxacin , Moxifloxacin, Tetracycline, Doxycycline, Azithromycin Trimethoprim/Sulfamethoxazole, , and Chloramphenicol (P-value <0.05).(figure 2)

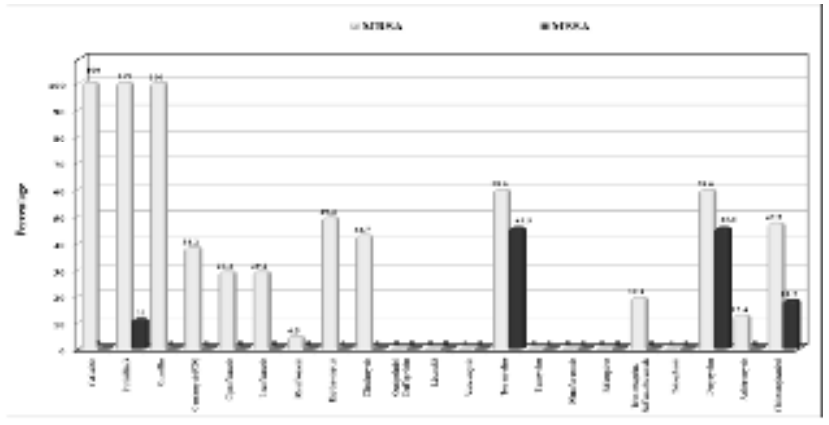

Figure (2): Comparison between MRSA and MSSA regarding antibiotics sensitivity.

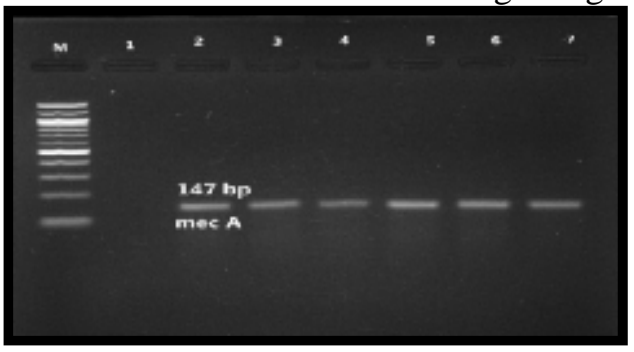

Figure (3): Electrophoresis of PCR products with primers for mec A. Lane M, 100 bp molecular weight marker; from lane 2,to lane 7, 147-bp bands from mec A positive samples; lane 1, negative control.

\section{Discussion}

In this study 350 pus samples were collected from patients presented with wound infection admitted at Sohag University Hospitals. Samples were cultured on mannitol salt agar and Staphylococcus aureus was diagnosed in 100 patients $(28.7 \%)$ and confirmed by microscopic examination showing gram positive cocci arranged in grape like clusters. All the 100 samples were coagulase positive .This was in agreement with many other studies to which reported that $S$. aureus was the predominant microorganism (40-60\%) of the total microorganisms isolated from different types of 
wounds (Santosaningsih et al., 2018)[9] (Bessa et al., 2015) [10].In a studyof Pires et al.( 2018) [11], S. aureus was detected in $82.9 \%$ of infected wounds.

S.aureus isolates were highly resistant to Cefoxitin and Oxacillin. Among the 100 isolated S.aureus strains,89(89\%) were identified as MRSA by cefoxitin disc diffusion method., confirmed by vitek2 automated system and by detection of mec A gene by PCR. Prevalence of MRSA was high also in a study of John and Murugan (2014)[12] (81\%).This percentage is higher than the studies of Neopane et al. (2018) (30\%)[13] and Santosaningsih et al.(2018) [9](3.1\%) The high incidence of MRSA wound infections in the hospital may be due to overcrowding of the healthcare facility, unhindered access of patients' relatives to the patients and low healthcare personnel-patient ratio, which can all facilitate nosocomial transmission of the infection in the hospital. attributed to the fact that MRSA is usually spread by direct contact via infected wound samples or contaminated hands of the health care providers

All S.aureus isolates were resistant to Penicillin .This is agreed with many studies which reported that more than $80 \%$ of isolated S.aureus were resistant to penicillin Neopane et al. (2018) [13], Olowo-Okere et al. (2017) [14] and Belbase et al. (2017) [15]. Most strains of S.aureus are now resistant to penicillin as they contain penicillinase, which is a specific type of $\beta$-lactamase. Our S.aueus isolates were moderately resistant to Gentamycin(34\%), Erythromycin(44\%),

Clindamycin(38\%) .These results are in coordenence with the Neopane et al. (2018) [13] who found that (34\%) of S.aureus isolates were resistant to Gentamycin, (35\%) to Erythromycin $(35 \%)$ to and Clindamycin(26.2\%) and Belbase et al.(2017) [15],who reported that S.aureus isolates were also moderately resistant to Gentamycin (31.5\%), Erythromycin $(55.5 \%)$ but resistance to clindamycin was lower than our study (11\%). OlowoOker et al.(2017) [14], reported higher rate of resistance to Erythromycin(100\%) and gentamycin $(85 \%)$.

Also S.aureus isolates were moderately resistant to Tetracycline(54\%), Doxycycline $(58 \%)$ and Chloramphenicol(44\%).Roy et al. (2017) [16] reported also moderate rate of resistance of S.aureus to tetracycline (42\%). Neopane et al. (2018) [13], and Belbase et al.(2017) [15], reported lower resistance pattern of isolated S.aueus to Tetracycline (1\% and $1.5 \%$ respectively)and Chloramphenicol( $3 \%$ and $8 \%$ respectively).

S.aureusisolates showed similar resistance pattern to Ciprofloxacin and Levofloxacin (26\%) and low resistance to Moxifloxacin(4\%).This results are in agreement with a studyperformed by Onwubiko and Sadiq (2011) [17] who reported that $31 \%$ of isolated s.aureus were resistant to ciprofloxacin. Neopane et al. (2018) [13] Belbase et al. (2017)[15] and OlowoOkere et al. (2017) [14] showed higher rate of resistance of S.aureus isolates to ciprofloxacin $(48 \%, 72 \%$ and $\quad 70 \%$ respectively).No resistance of S.aureus isolates to levofloxacin was reported by Trogan et al. (2016) [18] and Onwubiko and Sadiq (2011) [17]. Results of Gade and Qazi (2013) [19] study were in coordenence to our study regarding resistance of isolated S.aureus to levofloxacin $(30 \%)$ but showed higher resistance to moxifloxcin (21\%). Imran et al. (2011) [20] showed also high sensitivity of S.aureus to moxifloxain $(0 \%)$.

All S.aureus isolates were sensitive to Qunipristin/Dalfopristin, Linezolid, Vancomycin ,Tigecycline, 
Nitrofurantoin, Rifampicin and Teicoplanin. Most studies reported no or low resistance of S.aureus of to these antibiotics .Belbase et al. (2017) [14] and Neopane et al., (2018) [13]. Low resistance of S.aureus isolates has been reported by Bukhari et al.(2011) [21] toLinezolid (3.4\%), Vancomycin (1\%), Nitrofuratoin(4.2\%),Rifampicin (2\%)and Teicoplanin (2\%). Resultsof a study of Gitau et al. (2018) [22] were consistent with our results as they reported $2 \%$ resistance to Nitrofuratoin and Tigcycline, 3\% to Linezolid and Teicoplanin ,5\% to Vancomycin and $8 \%$ to Rifampicin .Roy et al. (2017) [16] and Rahim et al.(2016) [23] reported higher resistance rate to Vancomycin $(24.1 \%)$ and $(26.6 \%)$ respectively.

The difference in antibiotic susceptibility profile isolated S.aueus in different studies might be due to the difference in implementation of rigid infection control precautions ,the use of antibiotics, which may vary from one hospital to the other, duration of the studies, healthcare facilities and hygiene measures .

In comparison between MRSA and $M S S A$ regarding antibiotic susceptibility profile; All MRSA and MSSA isolates were sensitive to Vancomycin, Linezolid, Nitrofurantoin, Rifampicin, Tigecycline Qunipristin/Dalfopristin, and Teicoplanin .Also, all MRSA and MSSA isolates were resistant to penicillin. There was no significant difference between MRSA and MSSA in susceptibility to Ciprofloxacin ,Levofloxacin ,Moxifloxacin, Tetracycline ,Trimethoprim/Sulfamethoxazole,

Doxycycline, Azithromycin and Chloramphenicol (P value is more than 0.05). MRSA showed significantly higher resistance to gentamycin, erythromycin and clindamycin than $\operatorname{MSSA}(\boldsymbol{P}$ value is $<0.05$ ).

Yousefi et al. (2016) [24] also reported that all MRSA and MSSA isolates were sensitive to Qunipristin/Dalfopristin and Linezolid and resistant to penicillin . They found a statistically significant difference between susceptibility of MSSA and MRSA isolates toVancomycin, Nitrofurantoin, Rifampicin, Teicoplanin, Ciprofloxacin ,Levofloxacin, Tetracycline ,Trimethoprim/Sulfamethoxazole, Doxycycline and Chloramphenicol (P value is $<0.05$ ).

In study conducted by Akpaka et al.(2017) [5], there was a statistically significant difference between susceptibility of MSSA and MRSA isolates to Vancomycin, Linezolid, Nitrofurantoin, Rifampicin, Tigecycline ,Qunipristin/Dalfopristin, , Teicoplanin, Ciprofloxacin, Levofloxacin ,Moxifloxacin, tetracycline ,Trimethoprim/Sulfamethoxazole,

Doxycycline, Azithromycin and Chloramphenicol (P value is <0.05).In agreement to current study all MSSA and MSSA isolates were resistant to Penicillin.

No difference in susceptibility to Penicillin and Nitrofurantoin . All isolates were sensitive to Chloramphenicol Linezolid and Vancomycin.Such difference could be due to the difference in resistance patterns of $S$. aureus in different patient groups, hospitals, time periods and geographical locations.

The findings of this study underscore the need to strengthen infection control measures and improve surveillance of antibiotic resistant bacteria particularly $S$. aureus in the hospital as MRSA has been implicated in both nosocomial and community-onset infections which has shown increasing endemic and epidemic spread in the last four 
decades [23]. Further exacerbating the problem is the increasing multidrug resistant phenotype of many isolates of $S$. aureus, thereby limiting therapeutic options available for the management of its infections. Since patient-topatient transmission in healthcare settings, usually via hands of healthcare personnel has been demonstrated to be responsible for increase in incidence and prevalence of MRSA infections and those due to other multidrug resistant organisms, the first and the most effective means of curbing further transmission in healthcare setting is breaking the chain of transmission through proper hand hygiene, use of personal protective equipment (PPE), such as disposable gloves, isolation gown, face masks, goggles, face shields, etc. [23]. This will help to protect mucous membranes, airways, skin, and clothing from contact with infectious agents thereby preventing dissemination of resistant organisms from personnel to patients. Another factor identified for increase prevalence of MRSA and other resistant bacteria infections is the inappropriate use of broad-spectrum antibiotics [9]. Therefore, chemotherapy should be guided by sensitivity of the probable causative organism.

Lastly, alongside the education and training of medical and other healthcare personnel, should be created about the risk factors and routes of transmission of infection in the community and the healthcare settings [23]. In order to have a better understanding of the incidence and susceptibility of $S$. aureus in the hospital, an expanded study covering all clinical specimens in all service units in the hospital over an extended period of time should be conducted. Also, effort should be made in future studies to investigate the molecular basis underlying resistance of $S$. aureus in the hospital as data is currently unavailable on this in the hospital.

\section{Conclusion}

In the light of the findings of this study, it can be concluded that $S$. aureus is a major agent of wound infection at Sohag University Hospitals. The $S$. aureus isolates exhibited resistance to most of the antibiotics with the exception of Qunipristin/Dalfopristin, Linezolid, Vancomycin ,Tigecycline, Nitrofurantoin, Rifampicin and Teicoplanin. A high proportion of the $S$. aureus isolates were MRSA.

\section{References:}

1. Taddesse, Z., Tiruneh, M., \& Gizachew, M. (2014). Staphylococcus aureus and its Antimicrobial Susceptibility Pattern in Patients, Nasal carriage of Health Personnel, and objects at Dessie referral hospital, Northern Ethiopia. Global Journal of Medical Research.

2. Azeez-Akande, O. (2010). Global trend of methicillin-resistant Staphlococcus aureus and emerging challenges for control. African Journal of Clinical and Experimental Microbiology, 11(3).

3. Tong, S. Y., Davis, J. S., Eichenberger, E., Holland, T. L., \& Fowler, V. G. (2015). Staphylococcus aureus infections: epidemiology, pathophysiology, clinical manifestations, and management. Clinical microbiology reviews, 28(3), 603-661.

4. Taylor, T. A., \& Unakal, C. G. (2017). Staphylococcus Aureus.

5. Akpaka, P. E., Roberts, R., \& Monecke, S. (2017). Molecular characterization of antimicrobial resistance genes against Staphylococcus aureus isolates from Trinidad and Tobago. Journal of infection and public health, 10(3), 316323.

6. Anstead, G. M., Cadena, J., \& Javeri, H. (2014). Treatment of infections due to resistant Staphylococcus aureus. In Methicillin-Resistant Staphylococcus 
Aureus (MRSA) Protocols (pp. 259309). Humana Press, Totowa, NJ.

7. Kotz, P., Fisher, J., McCluskey, P., Hartwell, S. D., \& Dharma, H. (2009). Use of a new silver barrier dressing, ALLEVYN? Ag in exuding chronic wounds. International wound journal, 6(3), 186-194.

8. Zhang, K., McClure, J. A., Elsayed, S., Louie, T., \& Conly, J. M. (2005). Novel multiplex PCR assay for characterization and concomitant subtyping of staphylococcal cassette chromosome mec types $\mathrm{I}$ to $\mathrm{V}$ in methicillin-resistant Staphylococcus aureus. Journal of clinical microbiology, 43(10), 5026-5033.

9. Santosaningsih, D., Santoso, S., Setijowati, N., Rasyid, H. A., Budayanti, N. S., Suata, K., ... \& Prakoeswa, C. R. (2018). Prevalence and characterisation of Staphylococcus aureus causing community-acquired skin and soft tissue infections on Java and Bali, Indonesia. Tropical Medicine \& International Health, 23(1), 34-44.

10.Bessa, L. J., Fazii, P., Di Giulio, M., \& Cellini, L. (2015). Bacterial isolates from infected wounds and their antibiotic susceptibility pattern: some remarks about wound infection. International wound journal, 12(1), 47-52.

11.Pires, B. M. F. B., Fuly, P. D. S. C., Ferreira-Carvalho, B. T., \& Teixeira, L. A. (2018). Monitoring and Molecular Characterization of Staphylococcus aureus Isolated From Chronic Wounds. Advances in skin \& wound care.

12.John, N. P., \& Murugan, S. (2014). Biofilm formation by methicillin resistant Staphylococcus aureus and their antibiotic susceptibility pattern: An in vitro study. Current research in bacteriology, 7(1), 1-11.

13. Neopane, P., Nepal, H. P., Shrestha, R., Uehara, O., \& Abiko, Y. (2018). In vitro biofilm formation by Staphylococcus aureus isolated from wounds of hospital-admitted patients and their association with antimicrobial resistance. International Journal of General Medicine, 11, 25.
14.Olowo-Okere, A., Atata, R. F., Abass, A., Shuaibu, A. S., Yahya, U. H., \& Tanko, N. (2017). Incidence and Antibiotic Susceptibility Profile of Staphylococcus aureus Isolates from Wounds of Patients at Specialist Hospital, Sokoto, Nigeria. Journal of Medical Bacteriology, 6(3, 4), 44-50.

15.Belbase, A., Pant, N. D., Nepal, K., Neupane, B., Baidhya, R., Baidya, R., \& Lekhak, B. (2017). Antibiotic resistance and biofilm production among the strains of Staphylococcusaureus isolated from pus/wound swab samples in a tertiary care hospital in Nepal. Annals of clinical microbiology and antimicrobials, 16(1), 15.

16.Roy, S., Ahmed, M. U., Uddin, B. M. M., Ratan, Z. A., Rajawat, M., Mehta, V., \& Zaman, S. B. (2017). Evaluation of antibiotic susceptibility in wound infections: A pilot study from Bangladesh. F1000Research, 6.

17. Onwubiko, N. E., \& Sadiq, N. M. (2011). Antibiotic sensitivity pattern of Staphylococcus aureus from clinical isolates in a tertiary health institution in Kano, Northwestern Nigeria. Pan African Medical Journal, 8(1).

18.Trojan, R., Razdan, L., \& Singh, N. (2016). Antibiotic susceptibility patterns of bacterial isolates from pus samples in a tertiary care hospital of Punjab, India. International journal ofMicrobiology, 2016.

19.Gade, N. D., \& Qazi, M. S. (2013). Fluoroquinolone therapy in Staphylococcus aureus infections: where do we stand?. Journal of Laboratory physicians, 5(2), 109.

20.Imran, M., Faheem, M., Aslam, V., Hakeem, A., \& Shah, A. (2011). Wound infections and culture sensitivity pattern in pediatric burn patients. Journal of Postgraduate Medical Institute (PeshawarPakistan), 23(4).

21.Bukhari, S. Z., Ahmed, S., \& Zia, N. (2011). Antimicrobial susceptibility pattern of Staphylococcus aureus on clinical isolates and efficacy of laboratory tests to diagnose MRSA: a multi-centre study. Journal of Ayub 
Medical College Abbottabad,23(1), 139-142

22.Gitau, W., Masika, M., Musyoki, M., Museve, B., \& Mutwiri, T. (2018). Antimicrobial susceptibility pattern of Staphylococcus aureus isolates from clinical specimens at Kenyatta National Hospital. BMC research notes, 11(1), 226.

23.Rahim, F., Ullah, F., Ishfaq, M., Afridi, A. K., ur Rahman, S., \& Rahman, H. (2016). Frequency of common bacteria and their antibiotic sensitivity pattern in diabetics presenting with foot ulcer. Journal of Ayub Medical College Abbottabad, 28(3), 528-533.

24. Yousefi, M., Pourmand, M. R., Fallah, F., Hashemi, A., Mashhadi, R., \& Nazari-Alam, A. (2016). Characterization of Staphylococcus aureus biofilm formation in urinary tract infection. Iranian journal of public health, 45(4), 485. 